\title{
CAIPIRINHA-accelerated 2D bSSFP imaging with improved banding behavior using Gradient- Controlled Local Larmor Frequency (GC-LOLA)
}

\author{
Peter Speier ${ }^{1 *}$, Daniel Staeb ${ }^{3,2}$, Edgar Mueller $^{1}$ \\ From 19th Annual SCMR Scientific Sessions \\ Los Angeles, CA, USA. 27-30 January 2016
}

\section{Background}

Cardiac MRI often requires a careful trade-off between SNR, spatio-temporal resolution and slice coverage. Providing fast acquisitions, high SNR and flow/motion robustness, bSSFP has become the dominant sequence. Drawbacks are high SAR levels and sensitivity to B0 inhomogeneities. For improving slice coverage, MSCAIPIRINHA [1] has emerged as a standard method. By simultaneously scanning multiple slices, it provides acceleration in slice direction with minimal SNR penalty.

When combining MS-CAIPIRINHA with bSSFP, the sequence steady-state and contrast have to be maintained. Two methods are available: The first [2] employs RFbased multi-slice encoding [1], generating slice-specific shifts in the bSSFP pass-band structure effectively reducing the off-resonance robustness by a factor of two. The second [3] applies balanced gradient encoding during readout, potentially increasing the sensitivity to eddy currents for small inter-slice distances because encoding changes from excitation to excitation.

\section{Methods}

We propose a new method, called gradient-controlled local larmor adjustment (GC-LOLA), that eliminates the drawback of the RF-encoded combination in two steps: (1) By slightly unbalancing the slice select gradient, the Larmor frequency is made locally dependent, which compensates the relative shift between the pass-bands. (2) In addition, the RF phase cycles are modified to shift the centers of the aligned pass-bands to resonance. The method is illustrated in Figure 1 for two slices S0 and $\mathrm{S} 1$ at slice positions D0 and D1. The pass-band shifts

${ }^{1}$ Siemens Healthcare GmbH, Erlangen, Germany

Full list of author information is available at the end of the article

are fully corrected by unbalancing the slice gradient by the moment $\mathbf{M}$, distributed evenly on slice pre- and rephaser, and subtracting the residual off-resonance $\boldsymbol{\Phi}_{\mathrm{G}}$ from the RF phase increments in both slices. To test the concept, phantom and in-vivo measurements were performed using a bSSFP sequence prototype, modified inhouse to support MS-CAIPIRINHA and GC-LOLA (MAGNETOM Aera and Skyra, Siemens Healthcare $\mathrm{GmbH}$, Erlangen).

\section{Results}

The Phantom results (Figure 2 top row, slice thickness $5 \mathrm{~mm}$, slice positions S0: $55 \mathrm{~mm}, \mathrm{~S} 1: 70 \mathrm{~mm}$, flip $40^{\circ}$ ) demonstrate the successful restoration of the original band pattern. Due to the gradient unbalancing, the stop-bands appear slightly blurred. The benefit of increasing the offresonance robustness can be seen from the volunteer scan (Figure 2 bottom row, slice thickness $5 \mathrm{~mm}$, gap 100\%, TR $2.9 \mathrm{~ms}$, TE $1.3 \mathrm{~ms}$ ): A stop-band is shifted out of the posterior of the left ventricle (LV) and the signal in the LV blood pool is more homogeneous.

\section{Conclusions}

Our preliminary results indicate that GC-LOLA stabilizes MS-CAIPIRINHA-accelerated bSSFP with respect to field inhomogeneities, without the need for toggled gradients from TR to TR.

\section{Authors' details}

${ }^{1}$ Siemens Healthcare GmbH, Erlangen, Germany. ${ }^{2}$ Department of Diagnostic and Interventional Radiology, University of Wuerzburg, Wuerzburg, Germany. ${ }^{3}$ Centre for Advanced Imaging, The University of Queensland, Brisbane, QLD, Australia. 


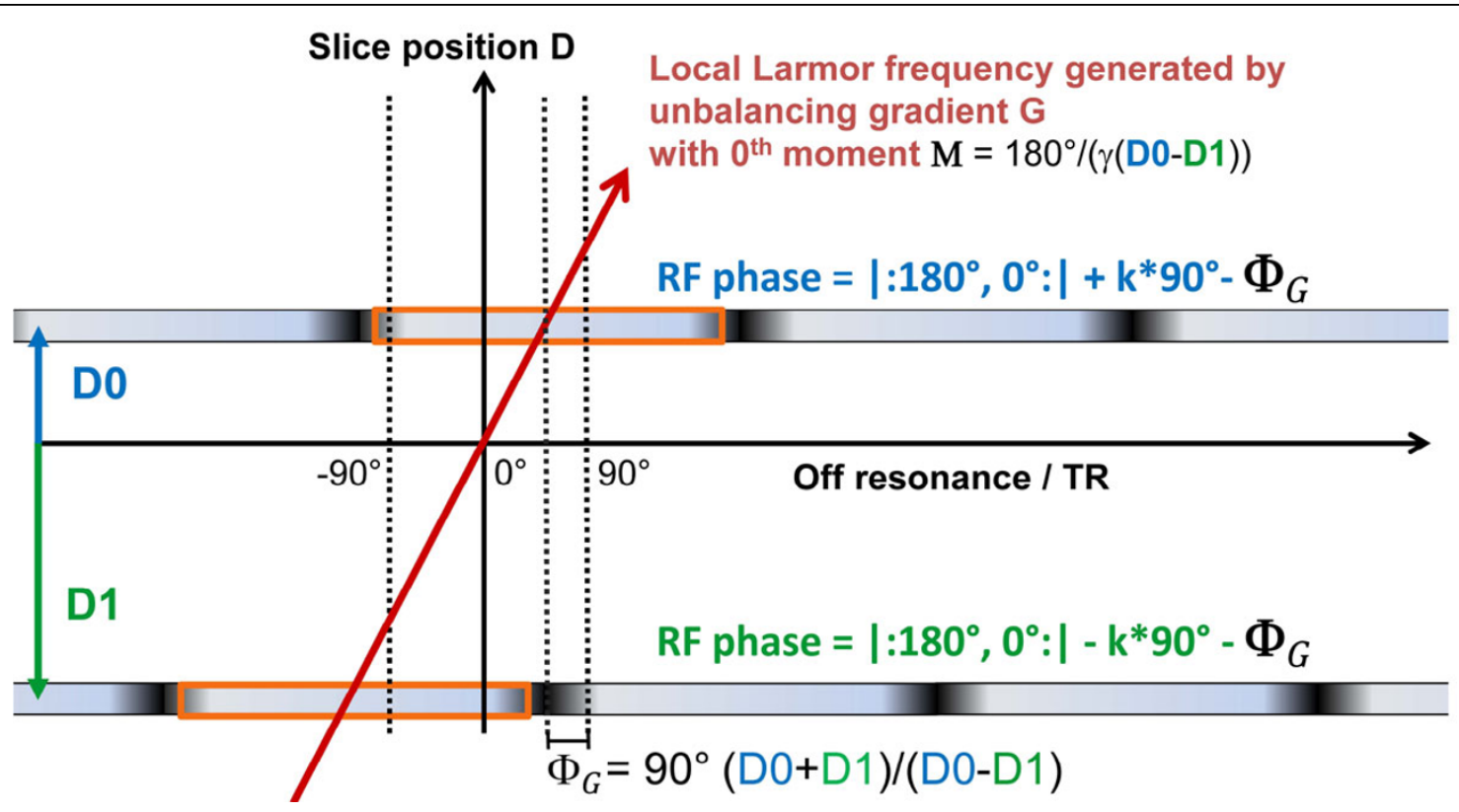

Figure 1 bSSFP with GC-LOLA for two slices.
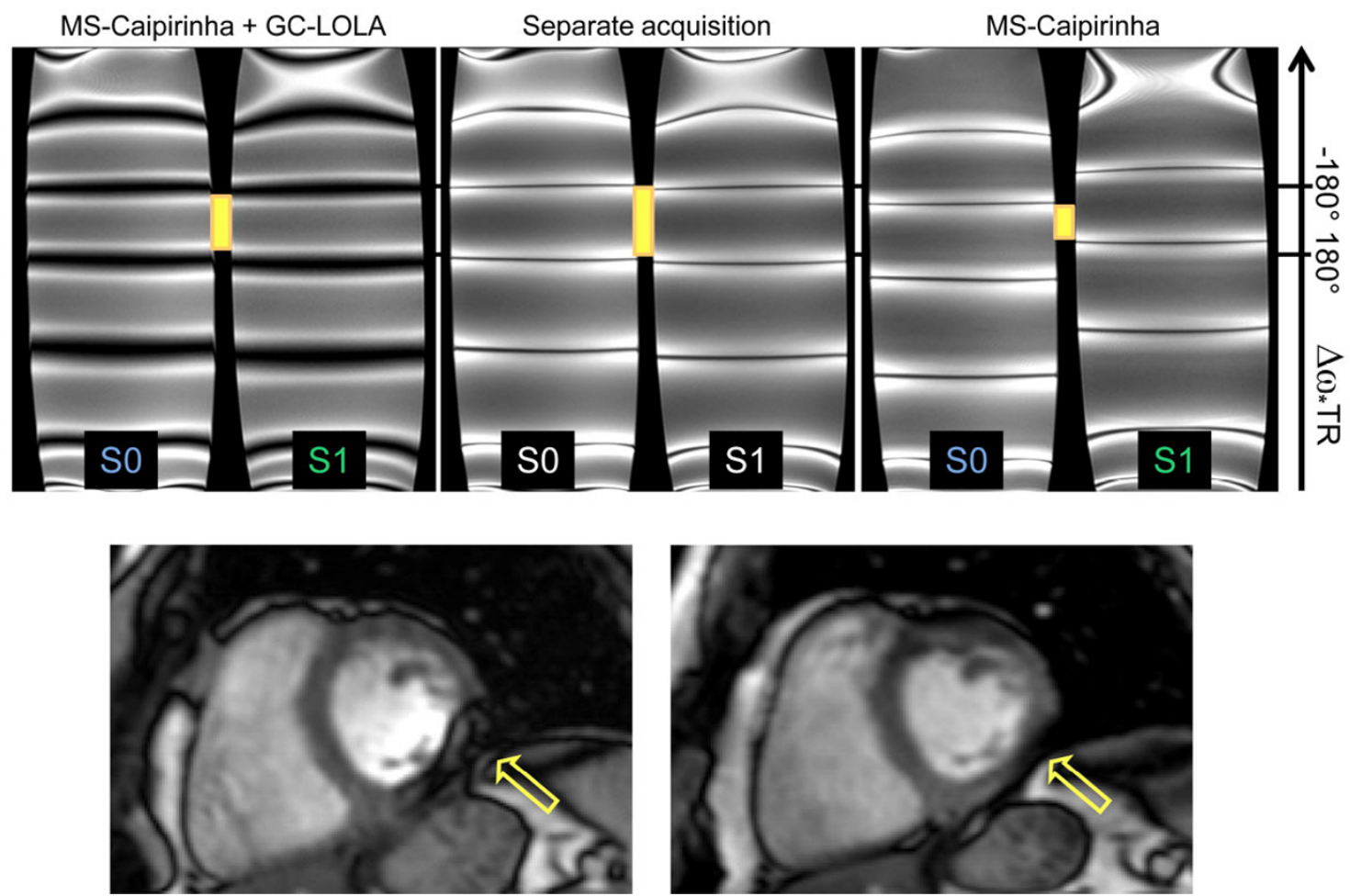

Figure 2 Top row: bSSFP band structure in a phantom with linear gradient for RF-encoded MS-CAIPIRINHA with two simultaneously excited slices without (left) and with GC-LOLA (right) compared to two single-slice acquisitions (center). Conventional MS-CAIPIRINHA shifts the stop-bands by $+1 / 4$ and $-1 / 4$ of the band distance in slice 1 and 2, respectively. The application of GC-LOLA shifts the stop-bands back to their original positions and blurs them. The common central pass-band is indicated with orange bars. Bottom row: One of two slices of an MSCAIPIRINHA scan of a healthy volunteer at 3T without (left) and with (right) GC-LOLA. The stop-band indicated by the arrow has successfully been moved out of the posterior wall. 
Published: 27 January 2016

\section{References}

1. Breuer: MRM 2005, 53:684-691.

2. Stäb : MRM 2011, 65:157-164.

3. Duerk : 2013, US2013/0271128 A1.

doi:10.1186/1532-429X-18-S1-P301

Cite this article as: Speier et al:: CAIPIRINHA-accelerated 2D bSSFP

imaging with improved banding behavior using Gradient-Controlled

Local Larmor Frequency (GC-LOLA). Journal of Cardiovascular Magnetic

Resonance 2016 18(Suppl 1):P301.

Submit your next manuscript to BioMed Central and take full advantage of:

- Convenient online submission

- Thorough peer review

- No space constraints or color figure charges

- Immediate publication on acceptance

- Inclusion in PubMed, CAS, Scopus and Google Scholar

- Research which is freely available for redistribution

Submit your manuscript at 